\title{
Erratum to: Artificial Intelligence and Mobile Services - AIMS 2018
}

Marco Aiello, Yujiu Yang, Yuexian Zou, and Liang-Jie Zhang

\author{
Erratum to: \\ M. Aiello et al. (Eds.): Artificial Intelligence and Mobile \\ Services - AIMS 2018, LNCS 10970, \\ https://doi.org/10.1007/978-3-319-94361-9
}

The original version of the book frontmatter has been revised. Yujiu Yang and Liang-Jie Zhang affiliations have been corrected.

The updated online version of this book can be found at https://doi.org/10.1007/978-3-319-94361-9

(C) Springer International Publishing AG, part of Springer Nature 2018 M. Aiello et al. (Eds.): AIMS 2018, LNCS 10970, p. E1, 2018.

https://doi.org/10.1007/978-3-319-94361-9_21 